\title{
Spatial dynamics of confined semiconductor microcavity polaritons
}

\author{
R. Cerna, ${ }^{1, *}$ T. K. Paraïso, ${ }^{1}$ Y. Léger, ${ }^{1}$ M. Wouters, ${ }^{2}$ F. Morier-Genoud, ${ }^{1}$ M. T. Portella-Oberli, ${ }^{1}$ and B. Deveaud-Plédran ${ }^{1}$ \\ ${ }^{1}$ Institut de Photonique et d'Électronique Quantiques, École Polytechnique Fédérale de Lausanne (EPFL), \\ CH-1015 Lausanne, Switzerland \\ ${ }^{2}$ Institute of Theoretical Physics, Ecole Polytechnique Fédérale de Lausanne (EPFL), CH-1015 Lausanne, Switzerland
}

(Received 19 December 2009; published 24 March 2010)

\begin{abstract}
We report on the dynamics of confined zero-dimensional polaritons. We excite resonantly discrete polariton states optically with a tailored picosecond laser pulse and observe their emission time resolved in the twodimensional $k$ space or real space. We are able to distinguish between three regimes. When the laser excites only one state no dynamics are observed. When a small number of well separated states are excited, the dynamics are described in terms of interference. When a quasicontinuum of states is excited, the dynamics are described in terms of ballistically propagating two-dimensional (2D) polaritons which scatter elastically at the potential barriers. The measured propagation velocities are reproduced with the theory of 2D polaritons, which sustains our interpretation.
\end{abstract}

DOI: 10.1103/PhysRevB.81.113306

PACS number(s): 78.55.Cr, 71.36.+c, 71.35.-y

Exciton polaritons are quasiparticles resulting from the strong coupling between excitons and photons. Due to their mixed light and matter nature they posses some very interesting properties such as bosonic statistics, ${ }^{1}$ very small effective mass due to their photonic component, and strong nonlinearities due to their excitonic part. ${ }^{2}$ Thanks to their small mass it is sufficient to confine polaritons within micrometer sized traps to produce an atomlike spectrum with discrete energy levels in contrast to the nanometer sized traps, which are necessary to confine bare excitons. This large scale as well as the optical accessibility of the wave function of polaritons via their photonic part offers a great possibility of controlling fundamental electronic excitations in a solid. ${ }^{3}$ This holds great promises for possible applications and indeed various kinds of traps for creating zero-dimensional polaritons have been realized in the past few years by different groups. $^{4-7}$

So far there is no report about spatial dynamics of polaritons confined in three dimensions. Polariton dynamics in the linear regime have been mostly studied on two-dimensional (2D) microcavity polaritons. Due to cavity imperfections the majority of these studies have evidenced scattering on disordered static potentials. This kind of scattering is energy conserving and is known as resonant Rayleigh scattering (RRS). ${ }^{8,9}$ Ballistic propagation of polaritons has been reported only in few works. ${ }^{10,11}$

In this Brief Report, we study the spatial dynamics of semiconductor microcavity polaritons confined in all three dimensions by traps with cylindrical symmetry. After optical excitation with a laser pulse we image the dynamics of the created polaritons with a picosecond time resolution both in the two-dimensional real and momentum space. We show that the dynamics strongly depend on the density of states of the confined polaritons. Three different regimes are studied: one excited state, few and well-separated excited states and a quasicontinuum of excited states. While in the first regime no dynamics are observed, the two other regimes show specific dynamics, which can be understood in different ways. When exciting a small number of well-separated states, the dynamics are described in the picture of coherent superposition of states. However when a quasicontinuum of states is excited, the temporal evolution of the polariton gas is better described in terms of ballistically propagating two-dimensional polaritons which undergo elastic geometrical scattering at the potential barrier. This assumption is proved by extracting the polariton velocities for different in-plane momenta. A decrease in velocity at large $k$ values shows a striking difference between polariton and photon dynamics.

Our results demonstrate that by laterally confining polaritons in traps of sizes comparable to the typical photonic disorder scale, one can isolate the polaritons in an environment free of disorder. Long distance ballistic propagation can in this case be studied by using multiple elastic scattering inside the trap.

The investigated sample consists of a single semiconductor quantum well (QW) placed in the middle of a $\lambda$ microcavity (MC). The high quality $\mathrm{QW}$, which is composed of a 8-nm-thick $\operatorname{In}_{0.04} \mathrm{Ga}_{0.96}$ As layer sandwiched between two GaAs barrier layers, shows a sharp exciton resonance (500 $\mu \mathrm{eV}$ full width half maximum) at $\mathrm{E}_{\mathrm{x}}=1.484 \mathrm{eV}$. The two distributed Bragg reflectors (DBRs) of the MC are made of 21 (top) and 22 (bottom) layer pairs of AlAs/GaAs. The resonance energy of the cavity $\mathrm{E}_{c}=c h / \lambda_{c}$ is given by the cavity spacer thickness $\lambda_{c} / n$. The sample is wedge shaped thereby allowing to change the resonance wavelength $\lambda_{c}$ of the $\mathrm{MC}$ as a function of sampling position. Thus the energy detuning $\left(\delta=\mathrm{E}_{c}-\mathrm{E}_{x}\right)$ between the cavity photons and the QW excitons also changes with the position. Round mesas of different sizes $(3,9$, and $20 \mu \mathrm{m}$ in diameter) have been etched on the top of the spacer before growing the top DBR. In this way the resonance energy $\mathrm{E}_{c}=\hbar \omega_{c}$ of the cavity is locally lowered. The mesas have a height of $\Delta \mathrm{L}=6 \mathrm{~nm}$, which corresponds to a potential depth of about $9 \mathrm{meV}$ at the exciton energy. ${ }^{4}$ For a more complete description and characterization of the sample see also Refs. 12 and 13.

All experiments presented here were done in transmission with a photoluminescence setup. For the optical excitation of the polaritons we employed a femtosecond Ti:Sa laser and a pulse shaper which allowed us to shape narrowband pulses (300 $\mu \mathrm{eV}, 8 \mathrm{ps}$ ) at the frequencies we needed for resonant excitation. The laser beam was focused on the sample with a camera objective ( $f=55 \mathrm{~mm}$ and 1.2 aperture) providing an 


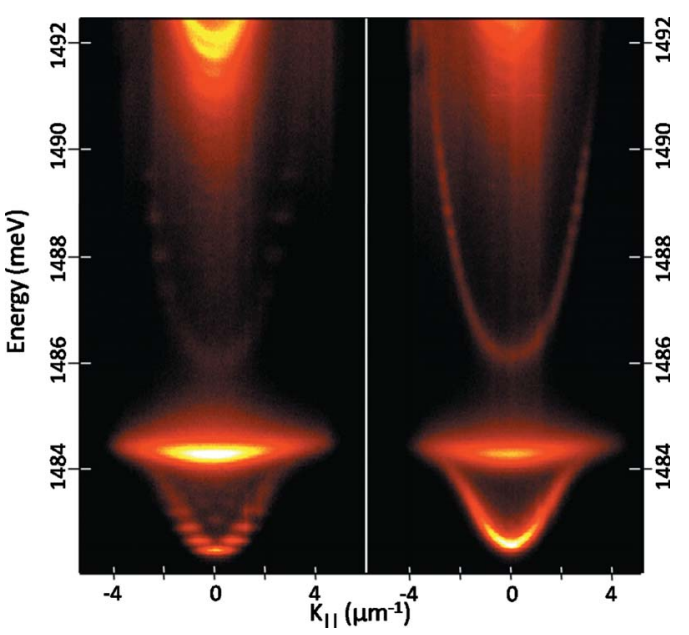

FIG. 1. (Color online) Polariton dispersion curves of a 9 (left side) and $20 \mu \mathrm{m}$ mesa (right side) around zero detuning. The sample was excited nonresonantly with a cw laser. In both cases one can clearly distinguish the lower and upper polariton branches (LP and UP) of the confined polaritons (1482-1484 and 1486-1490 $\mathrm{meV}$ ) as well as the LP and UP branches of the 2D microcavity polaritons from around the mesas (1484.5 meV and above 1490 $\mathrm{meV})$. One can clearly observe discrete polariton states in the $9 \mu \mathrm{m}$ mesa and rather a continuum of states in the $20 \mu \mathrm{m}$ mesa.

excitation spot of about $25 \mu \mathrm{m}$ for small excitation angles up to $30 \mu \mathrm{m}$ for larger excitation angles. In order to excite properly the polariton states we controlled the excitation incidence angle and matched the in-plane momentum $\mathbf{k}_{\|}$ $=\left(k_{x}, k_{y}\right)$ of the photons to the polariton states $\left(k_{\|}\right.$ $\left.\propto \sin (\theta) / \lambda_{\text {laser }}\right)$. On the detection side we employed a streak camera for time resolved imaging in $k$ and real space and a spectrometer. The streak camera we used has a time resolution of about 2 ps. By consecutively imaging adjacent slices of the real or the momentum space on the entrance slit of the streak camera we could obtain time-resolved twodimensional images of the excited polariton wave functions. The results presented here were all obtained in the linear regime.

The lateral confinement of the two-dimensional polaritons through the round mesas results in a discrete energy spectrum of zero-dimensional polaritons. ${ }^{4,13}$ The energy spacing between these confined states decreases with increasing diameter of the mesas and with increasing detuning. In this Brief Report, we kept the detuning always around zero and changed the density of states by investigating different sized mesas. This is demonstrated in Fig. 1, where we show the dispersion curves of a 9 and $20 \mu \mathrm{m}$ mesa around zero detuning. While we still observe well separated discrete states in the lower and upper polariton branch of the $9 \mu \mathrm{m}$ mesa we have a quasicontinuum of states in the $20 \mu \mathrm{m}$ mesa. The cylindrical symmetry of the trap allows us to label the discrete eigenstates with a radial quantum number $n$ and an angular quantum number $m$ with $n=1,2 \cdots$ and $m=0,1 \cdots$ (Ref. 14). The trap symmetry implies as well that eigenstates with $m \neq 0$ are twice degenerate with respect to $\pm m$. When exciting these states resonantly with a cw laser and observing their far field emission (which corresponds to the momentum probability distribution of the excited polaritons) one ob-

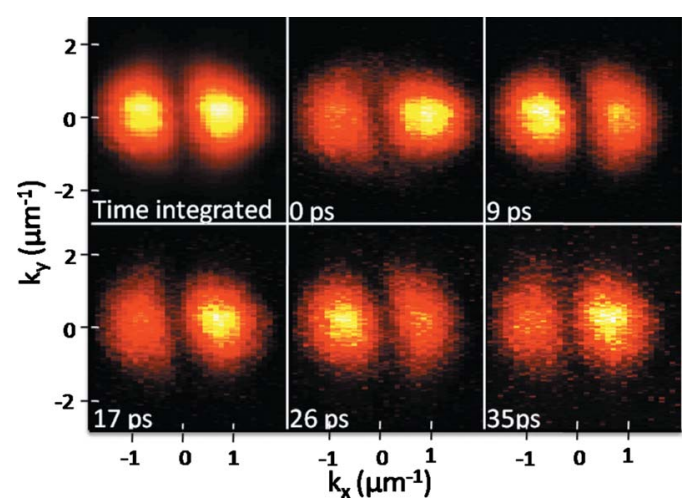

FIG. 2. (Color online) Time integrated and time resolved images of a $9 \mu \mathrm{m}$ mesa after resonant pulsed excitation. The pulse energy was tuned between the ground state $(1,0)$ and the first-excited state $(1,1)$. Since the in-plane momentum of the laser was about 0.8 $\mu \mathrm{m}^{-1}$ we excited more the first-excited state due to the better overlap with the laser. The time integrated image shows roughly the same pattern as under resonant $\mathrm{cw}$ excitation of $(1,1)$, while the time-resolved images reveal intensity beatings between the two lobes due to interference between the two-excited states.

serves a lobe pattern along the angular direction $\phi .{ }^{3}$ This comes from a phase locking effect which locks the phase of the excited $\pm m$ states to the phase of the laser. The different phase evolutions along the angular direction $\phi\left(\psi_{ \pm m}\right.$ $\propto e^{ \pm i m \phi}$ ) then give rise to the observed interference pattern. This phase locking effect can be used to manipulate the wave function of the excited states. ${ }^{3}$

Under $\mathrm{cw}$ excitation the observed coherent emission occurs at the energy of the laser and its phase remains locked to the phase of the laser. This is different under pulsed excitation. In this case the states which are resonant with the laser pulse are excited at their eigenenergies and their phases are only imposed by the laser during the pulse duration. This means that each exited state will emit at its eigenenergy with a phase that evolves freely. For $3 \mu \mathrm{m}$ mesas and for $9 \mu \mathrm{m}$ mesas at negative detuning it is possible to excite mainly one confined polariton state. In this case the time integrated images as well as all time-resolved images (for all delays where the emission is still visible) show the same pattern as under resonant $\mathrm{cw}$ excitation. The fact that the pattern does not get blurred shows us that the coherence time of the created polaritons is significantly longer than their lifetime.

Now, we are interested in the dynamics after the excitation of a small number of well separated states. We investigated this in a $9 \mu \mathrm{m}$ mesa by tuning the laser energy between two different states and by going toward higher $m$ states, where the energy spacing between the confined states becomes smaller. In this regime one observes different kind of dynamics, depending on the number of the excited states and on their quantum numbers. One example is shown in Fig. 2. In this experiment the pulse energy has been tuned between the ground $m=0$ and first $m= \pm 1$ excited state. The time integrated image shows a similar pattern as the one obtained under cw excitation, when exciting the first-excited state. The time-resolved images reveal however that there are beatings between the two observed lobes. The time period of the beatings corresponds to the energy spacing between the 


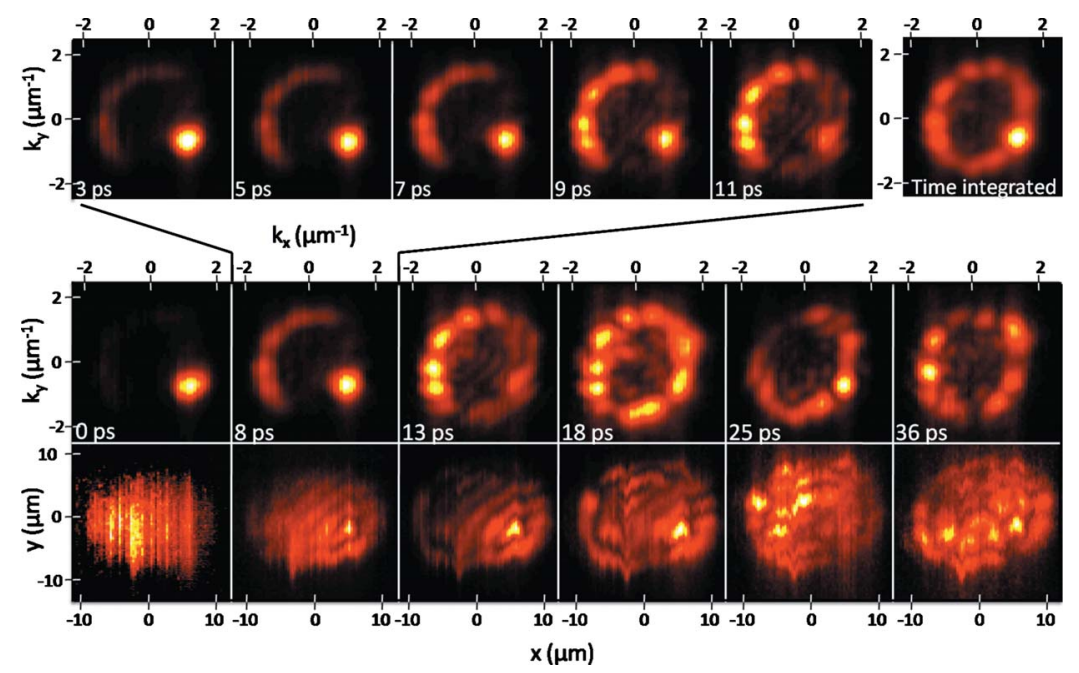

FIG. 3. (Color online) Time resolved $k$ space and the corresponding real space images of a $20 \mu \mathrm{m}$ mesa at negative detuning $(\delta$ $\approx-1 \mathrm{meV}$ ) after resonant pulsed excitation. Upper part: zoom showing the appearance of the back scattering signal and the build up of the lobes at early times. Lower part, first row: $K$-space images showing the buildup and disappearing of the forward and back scattering peak after excitation. Second row: corresponding real space images (same mesa, conditions, and times as first row) showing the polariton movements inside the mesa.

two excited states. This shows that we are observing an interference effect between these two states. The two lobes of the first-excited state have a phase shift of $\pi$, while the phase of the ground state is constant over the whole mesa. The phase relation between these states changes with a frequency defined by their energy splitting. This results in the out of phase periodic change from constructive to destructive interference of the two observed lobes. In general for $3 \mu \mathrm{m}$ mesas and for $9 \mu \mathrm{m}$ mesas at negative detuning it is possible to identify the excited states by dispersing their luminescence in a spectrometer. By taking into account the properties of these states, such as quantum numbers $(n, m)$ and energy spacing, it is then possible to explain the observed dynamics.

In $20 \mu \mathrm{m}$ mesas the energy spacing becomes of the same size as the linewidth of the eigenstates or even smaller and one cannot any longer speak of discrete eigenstates. In this case the laser pulse excites almost a continuum of states. The observed time integrated emission patterns in $k$ space as well as the dynamics are now much more complicated, as shown in Fig. 3. The observed lobes in $k$ space are much smaller than for the smaller mesas which are a sign of poorer confinement. We will show that, although the polaritons in the $20 \mu \mathrm{m}$ mesas feel the lateral confinement, their dynamics can very well be explained in terms of ballistically propagating two-dimensional polaritons.

Figure 3 shows time resolved 2D real and $k$-space images of the same mesa taken under the same conditions. The early times after excitation are illustrated separately in a zoom in the upper part of the figure. At the beginning one observes only one spot in the $k$-space images and in real space one observes the luminescence distributed over the whole mesa with a higher intensity in the middle. This reflects the Gaussian intensity profile of the laser. Polaritons are indeed created over the whole mesa with a precise in-plane momentum (which corresponds to a single propagation direction), given by the laser incidence angle. At later times ( $8 \mathrm{ps}$ ) one observes in $k$ space a blurred luminescence half-ring from the opposite direction. This half-ring comes from polaritons which were created close to the potential barrier of the mesa. These polaritons very soon undergo geometrical elastic scattering at the barrier. In real space one observes at the same time, that the luminescence comes mainly from the right half of the mesa. This means that most of the polaritons have already moved to this area and continue moving toward the right edge. One can also observe some fringes resulting from interference between the forward and the backward propagating polaritons. Afterwards (13 ps) one observes in $k$ space a lobe pattern in the back scattering half-ring. At the same time the forward scattering signal has almost disappeared. The build up of these back scattering peaks out of the blurred luminescence half-ring and the decreasing intensity of the forward scattering peak are shown in the temporal zoom in the upper part of Fig. 3. In real space one observes at 13 ps an agglomeration of polaritons at the right edge of the mesa. This shows that the maximum of the created polaritons has reached the potential barrier and due to the potential geometry most of the polaritons are scattered in the back direction. At 18 ps one observes in $k$ space a whole luminescence ring with lobes, which shows that some of the back-scattered polaritons have already reached the left edge of the mesa and undergo again geometrical elastic scattering at the potential barrier. In real space one can now observe luminescence from both sides of the mesa. Seven picoseconds later (25 ps) the back scattering signal has disappeared and one observes mainly the forward scattering peak. In real space one observes now the polaritons mainly in the left half of the mesa. This means that most of the polaritons have been scattered twice and are now again moving toward the right side of the mesa. At even later times (36 ps) one observes again a whole luminescence ring with lobes in the reciprocal space, which corresponds to an interference pattern over the whole mesa in real space. This comes from the round shape of the potential, which will randomly distribute the polaritons after few scattering events. The appearance of lobes in the $k$ space, which are also visible in the time integrated image (upper right corner of Fig. 3), originate from the fact that we are not dealing here with a true continuum of states, but only with a quasicontinuum, as described before, due to the lateral confinement of the polaritons. The created polaritons need a certain time to probe the whole confining potential of the trap until they reach a steady state. The time integrated $k$-space image with its luminescence ring and the enhanced emission in the back direction might resemble RRS, but in our case the circular shape comes only from the shape of the trap and 


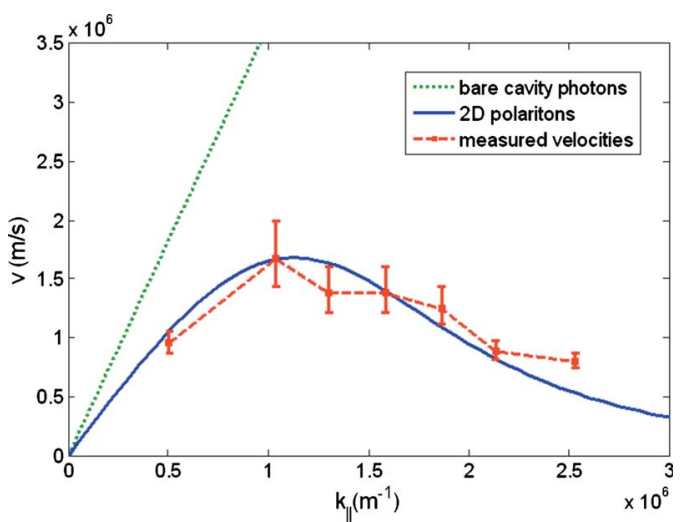

FIG. 4. (Color online) Measured polariton velocities (dashed line) inside a $20 \mu \mathrm{m}$ mesa as a function of in-plane momentum together with calculated velocities for two-dimensional photons (dotted line) and polaritons (solid line). The polariton velocity decreases with increasing momentum and is in general significantly lower than what one would expect from pure microcavity photons.

not from the interaction with the photonic disorder of the microcavity.

In order to sustain our interpretation of ballistically propagating polaritons, which undergo elastic geometrical scattering at the potential barriers, we have measured the propagating velocity of the created polaritons under resonant excitation in a $20 \mu \mathrm{m}$ mesa for different $k$ vectors. Knowing the mesa diameter and by extracting the appearance and disappearance times of the back and forward scattering peaks from the data we could estimate the polariton velocities. The results are shown in Fig. 4 (dashed line). It is important to note that the measured velocities do not increase continuously with $k$ and are significantly lower than the values one would expect for cavity photons (VCSELs and weak coupling regime for example). The dotted line in Fig. 4 represents the calculated in-plane group velocity for microcavity photons. It has been obtained by derivating the cavity dispersion curve at the given detuning. The pure cavity dispersion is given by the following expression:

$$
E_{c}\left(k_{\|}\right)=\sqrt{E_{c 0}^{2}+\hbar^{2} k_{\|}^{2} c^{2} / n_{e f f}^{2}},
$$

where $E_{c 0}$ is the resonance energy of the cavity at the investigated position and $n_{\text {eff }}$ is the effective refractive index of the cavity. The experimentally determined velocities can instead be fitted with a velocity curve (solid line in Fig. 4) calculated from the two-dimensional lower polariton dispersion curve

$$
E_{L P}\left(k_{\|}\right)=\frac{E_{X}\left(k_{\|}\right)+E_{c}\left(k_{\|}\right)}{2}-\frac{\sqrt{\left[E_{c}\left(k_{\|}\right)-E_{X}\left(k_{\|}\right)\right]^{2}+\Omega_{R}^{2}}}{2},
$$

where $\Omega_{R}$ is the Rabi splitting and $E_{X}\left(k_{\|}\right)=E_{X 0}$ $+\hbar^{2} k_{\|}^{2} /\left(2 M_{X}\right)$ is the exciton dispersion curve. We used the following experimentally determined parameters: $\Omega_{R}=3.6 \mathrm{meV}, \quad E_{X 0}=1484.35 \mathrm{meV}, \quad n_{\text {eff }}=3.3, \quad E_{c 0}$ $=1483.5 \mathrm{meV}$, and the exciton mass $M_{X}=0.3$ (free-electron mass).

The decreasing velocity for increasing momenta is hence explained by the peculiar shape of the polariton dispersion curve. The fact that the photon curve does not reproduce at all the observed velocities shows us that, even in the linear regime, the observed polariton behaviors are completely different than what one would expect from pure photons. The velocities reported here for ballistic propagating polaritons are also lower than what has been reported previously in Refs. 10 and 11. This comes from the fact that the other experiments were performed at strong negative detuning, where the polaritons are more photonlike.

In conclusion, we studied the dynamics of trapped zerodimensional polaritons after pulsed optical excitation for two different regimes. In the low density of states regime, where the energy splitting is larger than the natural linewidth, the dynamics are explained in terms of interference by identifying the excited eigenstates. In the high density of states regime, when exciting a high number of states, the dynamics can be explained in terms of ballistically propagating quasitwo-dimensional polaritons which undergo geometrical elastic scattering at the potential walls. The number and density of states can be controlled through the height and size of the mesas. Additionally one can also control the shape of the potential. This offers a possibility to overcome the photonic disorder of a microcavity sample and to design a homogenous environment for studying two-dimensional polariton dynamics. This holds as well great promises for possible applications where tailored spatial and temporal properties are needed.

The authors would like to thank V. Savona and G. Nardin for fruitful discussions. This work was supported by the Quantum Photonics NCCR and the Swiss National Science Foundation.

\footnotetext{
*roland.cerna@epfl.ch

${ }^{1}$ J. Kasprzak et al., Nature (London) 443, 409 (2006).

${ }^{2}$ M. Saba et al., Nature (London) 414, 731 (2001).

${ }^{3}$ R. Cerna et al., Phys. Rev. B 80, 121309(R) (2009).

${ }^{4}$ R. I. Kaitouni et al., Phys. Rev. B 74, 155311 (2006).

${ }^{5}$ D. Bajoni et al., Appl. Phys. Lett. 90, 051107 (2007).

${ }^{6}$ R. Balili et al., Science 316, 1007 (2007).

${ }^{7}$ C. W. Lai et al., Nature (London) 450, 529 (2007).
}

\footnotetext{
${ }^{8}$ R. Houdré et al., Phys. Rev. B 61, R13333 (2000).

${ }^{9}$ W. Langbein et al., Phys. Rev. Lett. 88, 047401 (2002).

${ }^{10}$ T. Freixanet et al., Phys. Rev. B 61, 7233 (2000).

${ }^{11}$ W. Langbein et al., Phys. Rev. B 75, 075323 (2007).

${ }^{12}$ O. El Daïf et al., Appl. Phys. Lett. 88, 061105 (2006).

${ }^{13}$ A. Baas et al., Phys. Status Solidi B 243, 2311 (2006).

${ }^{14}$ X. Leyronas and M. Combescot, Solid State Commun. 119, 631 (2001).
} 